\title{
Remote AI Supported E-Multidisciplinary Oncology Conference in Breast Cancer as a Technology and Method to Optimize Outcomes in the Peripheries
}

\author{
Constantinos S. MAMMAS ${ }^{\mathrm{a}, \mathrm{b}, 1}$, Adamantia S. MAMMA ${ }^{\mathrm{b}}$ \\ George PAPAXOINIS ${ }^{c}$, Irene GEORGIOU ${ }^{\mathrm{c}}$ \\ ${ }^{a}$ Elena El. Venizelou-Alexandra University Hospital of Athens, National \\ and Kapodistrian University of Athens (NKUA) \\ ${ }^{b}$ Program of Excellence 2014-16-Siemens Program for Greece \\ ${ }^{c}$ Saint Savvas Anticancer Hospital of Athens
}

\begin{abstract}
Aim: Feasibility-reliability control of Telemedicine Systems (TS) integrated with Multimedia Systems (MS) and Artificial intelligence (AI) for remote e-Multidisciplinary Oncology Conference in Breast Cancer. Material and Methods: Forty $(\mathrm{n} 1=40)$ patients suffering from breast surgical oncology malignant $(\mathrm{n} 2=32)$ and non-malignant $(\mathrm{n} 3=8)$ diseases classified to seven categories: Nipple Discharge, Dominant Breast Mass, Occult Breast Lesion, Early Breast Carcinoma, Advanced Breast Carcinoma, Recurrent Breast Carcinoma) and treated clinically with the standard diagnostic (Mammography, US, MRI, Cytology, Pathology, BRCA1/2 Mutation Predisposition and Breast Cancer Risk Analysis) surgical, auxiliary therapeutic methods. Then clinical decisions compared to those proposed remotely by the virtual AI supported e-Oncology Conference for each patient. Results: In four $(n 4=4)$ out of forty patients (TS, MS and $\mathrm{AI}$ ) supported decision making and surgical treatment proposal including postoperative Radiotherapy proposal was not as clear as expected. Non-output answer for non-malignant breast pathologies $(\mathrm{n} 3=8)$ was accurately indicated by (MS and AI). Mean accuracy of (TS, MS and AI) for: 1.Surgical Operative Planning including $\mathrm{Rad}=94.1 \%$, 2.Chem $=96.8 \%$, 3.Horm $=96.7 \% \quad[$ In $95 \%$, (Confidence interval: $85-99 \%$ )]. Conclusion: High feasibility-reliability of the virtual AI supported e-Multidisciplinary Oncology Conference for remote decision making and surgical planning and for optimum outcomes in Breast Cancer treatment makes it a clinical necessity especially for the periphery of Hellas.
\end{abstract}

Keywords. Breast Surgery, Tele-Radiology, Tele-Pathology, Tele-Cytology, AI, E-Multidisciplinary Oncology Conference

\section{Introduction}

The optimal management of patients with breast cancer $(\mathrm{BC})$ requires the expertise of specialists from different disciplines. This has led to the evolution of multidisciplinary teams (MDTs), allowing all key professionals to jointly discuss individual patients and

${ }^{1}$ Corresponding Author, Constantinos St. Mammas, MD, MSc, PhD, 9, Kononos Ave., Pagrati, Athens, Greece; Email: csmammas@med.uoa.gr. 
to contribute independently to clinical decisions. Also, it proved that MDTs for Multidisciplinary Oncology Conference in cooperation with Multidisciplinary Management of the breast cancer in high volume Breast Units optimize quality and have significantly better clinical outcomes for the benefit of the patients. However, MDTs for BC decision making in different regions in the periphery of Greece and in other countries are scarce [1,2]. The project searches feasibility-reliability of Telemedicine Systems (TS) integrated with Multimedia Systems (MS) and Artificial intelligence (AI) for remote e-Multidisciplinary Oncology Conference in Breast Cancer interrelated with Telepathology (TPE), Teleradiology (TRE) and Telecytology (TCE) remote evaluation $[3,4,5]$.

\section{Material and Methods}

Experimentation included the development of an OTE-TS similar Experimental TS (Exp.-TS) for the simulation of the integrated TS, MS and AI based TRE and TPE and TCE virtual examination of each patient (pn 1009078, 34931, 34932, 34933) (Table 1.).

Table 1. Comparison of the Modules between OTE-TS and Exp.-TS

\begin{tabular}{ccc}
\hline Modules & OTE-TS & EXP.-TS \\
\hline a. Medical record process & + & + \\
b. Examinations results. & + & + \\
c. Capture scanning and imaging. & + & + \\
d. DICOM and PACS vision. & + & + \\
e. Real-time tele-conference & + & + \\
f. Chat and whiteboard facilities. & + & + \\
g. Application sharing. & + & + \\
h. Tele-secretary facilities. & + & + \\
j. Tele-Mentoring facilities & + & + \\
i. Telecommunication net & ISDN based & Internet based \\
k. Multimedia System & + & + \\
1.AI computation system & - &
\end{tabular}

Simulation of the TRE upon N=40 Mammographic, MRI, CT, Breast and Upper Abdomen US digital images projected on the Exp.-TS in the internet (Cloud) for remote examination and decision making upon the virtual e-Multidisciplinary Breast Oncology Conference (Figure 1.). Simulation of the TPE including TCE based on the already worked out breast biopsies and their results for $\mathrm{N}=40$ digital microscopic images.

Integrated AI supported Decision Making (including clinical interventions and genetic consultation) and Surgical Planning analysis using SPSS (version 17.0).

Forty $(\mathrm{n} 1=40)$ patients suffering from breast surgical oncology malignant $(\mathrm{n} 2=32)$ and non-malignant $(\mathrm{n} 3=8)$ diseases classified to seven categories: Nipple Discharge, Dominant Breast Mass, Occult Breast Lesion, Early Breast Carcinoma, Advanced Breast Carcinoma, Recurrent Breast Carcinoma) and treated clinically with the standard diagnostic (Mammography, US, MRI, Cytology, Pathology, BRCA1/2 Mutation Predisposition and Breast Cancer Risk Analysis) surgical, auxiliary therapeutic methods. Then the diagnostic and therapeutic clinical decisions compared to those proposed by our (MS and AI) supported decision making and planning system and logic process for each patient. 


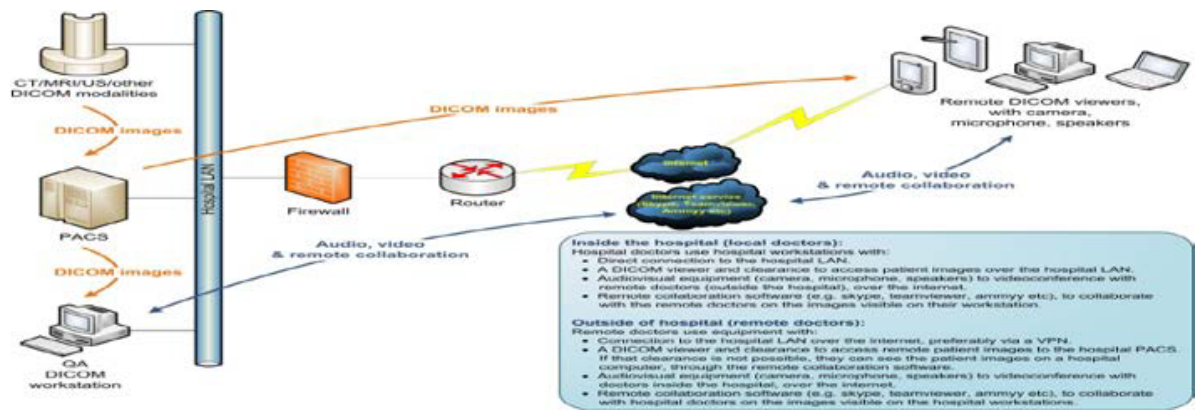

Figure 1. Simulation of the remote collaboration among specialists in the context of the remote virtual eMultidisciplinary Breast Oncology Conference via internet (PACS and DICOM based remote examination).

\section{Results}

In four (n4=4) out of forty $(\mathrm{n} 1=40)$ patients $(\mathrm{TS}, \mathrm{MS}$ and $\mathrm{AI})$ supported decision making and surgical treatment proposal including postoperative Radiotherapy proposal was not as clear as expected. Non-output answer for non-malignant breast pathologies (n3=8) was accurately indicated by (TS, MS and AI). Mean accuracy of (TS, MS and AI) for: 1.Surgical Operative Planning including $\mathrm{Rad}=94.1 \%$, 2.Chem $=96.8 \%$, 3.Horm $=96.7 \%$ [In 95\%, (Confidence interval: 85-99\%)] (Tables 2, 3, 4).

Table 2. Simulated TRE (Mammography, MRI, CT, Breast and Upper Abdomen US)

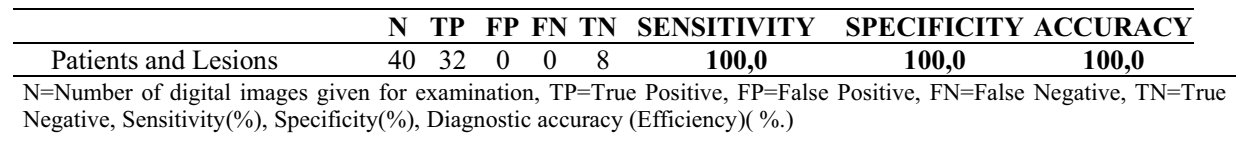

Table 3. Simulated TPE including TCE

\begin{tabular}{|c|c|c|c|c|c|c|}
\hline & $\mathbf{N}$ & TP & FP FN TN & SENSITIVITY & SPECIFICITY & ACCURACY \\
\hline Patients and Lesions & 40 & 32 & $\begin{array}{lll}0 & 0 & 8 \\
\end{array}$ & 100,0 & 100,0 & 100,0 \\
\hline
\end{tabular}

Table 4. AI supported Decision Making and Surgical Planning

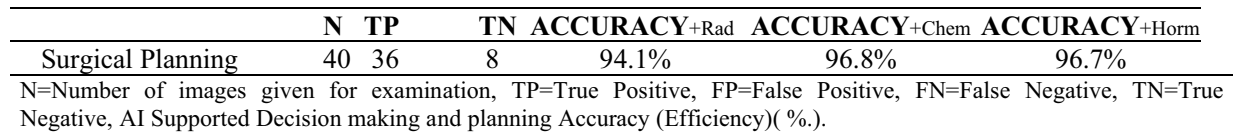

\section{Discussion}

Given the lack of Multidisciplinary Oncologic Conferences in the periphery of Greece and the fact that the clinical outcomes of the patients suffering from breast cancer are better under multidisciplinary management in high volume breast units the abovementioned results are very promising to realize virtual e-Multidisciplinary 
Oncology Conference to optimize quality of breast cancer management in the beginning. The results confirmed clinically in the periphery of Greece with a case of a female patient suffering from an exacerbation of right breast cancer (Extended locoregional right breast angio-sarcoma) which treated initially with chemotherapy (May 2021). Then treated with an additional chemotherapy schedule based on the decision of the remote e-Multidisciplinary Oncology Conference in Breast Cancer using the above described technology and method (TS, MS and AI from the General Hospital of Kalymnos, June 2021). With regard to safety issues Morgan [6] proposed the Virtual private networks (VPN) as adequate solutions for authentication, access control and confidentiality. The secure hypertext transfer protocol (https) can be used to encrypt for web distribution. A public key infrastructure (PKI) solves all the issues mentioned above. Clinical efficacy research protocols for further study of the remote eMultidisciplinary Oncology Conference in Breast Cancer is of high priority [7,8].

\section{Conclusion}

High feasibility-reliability of the virtual e-Multidisciplinary Oncology Conference in Breast Cancer integrated with AI supported decision making and treatment in Breast Cancer, makes it a clinical necessity for optimum clinical management and treatment of the breast cancer especially for the periphery of Hellas.

\section{References}

[1] Saini KS, Taylor C, Ramirez AJ, Palmieri C, Gunnarsson U, Schmoll HJ, et al. Role of the multidisciplinary team in breast cancer management: results from a large international survey involving 39 countries Annals of Oncol. 2012 Apr:23(4):853-9.

[2] Shao J, Rodrigues M, Corter AL, Baxter NN Multidisciplinary care of breast cancer patients: a scoping review of multidisciplinary styles, processes, and outcomes. Current Oncol. 2019 Jun;26 (3):385-397.

[3] Mammas CS, Mamma AS, Papaxoinis G, Georgiou I. AI supported decision making and planning in 40 breast surgery operations. In:Aristoteleio University of Thessaloniki Editor. Proceedings of the 32nd Panhellenic Congress of Surgery and International Forum; 2021 Jun 9-12;Thessaloniki:p.237.

[4] Mammas CS, Mandellos G, Economou GP, Lymberopoulos D. Structuring Expert-Leaded Medical Protocols for Telemedicine Systems. In: IEEE Editor. Proceedings of the 23rd Annual International Conference of the IEEE Engineering in Medicine and Biology Society; 2001 Oct 25-28; Constantinople: p.p.3529-32.

[5] Karavatselou E, Economou GP, Chassomeris C, Daneli V, Lymperopoulos D. OTE-TS-a new valueadded telematics service for telemedicine applications. IEEE Transactions on Information Technologies in Biomedicine. 2001 Sep;5(3):210-24.

[6] Morgan RH. Computer network security for the radiology enterprise Computer network. Radiology. 2001 Aug;220(2):303-09.

[7] Furness PN, Bamford WM. Tele-pathology. Curr Diag Pathol. 2001 Dec;7:281-291.

[8] Coiera E, editor. The Internet and Telemedicine. In: Coiera E, editor. Guide to Medical Informatics. 2nd ed. New York: CHM; c2007. 408 p. 